\section{The complex dynamics of diabetes modeled as a fractal complex-adaptive-system (FCAS)}

\section{A complexa dinâmica do diabetes modelado como um sistema fractal complexo adaptativo(FCAS)}

\section{Pierre Philippe}

Department of Social and Preventive Medicine

Faculty of Medicine, University of Montreal

M. d'Youville Bldg., PO Box. 6128, Station "A",

Montreal H3C 3J7, QC, Canada

Email: philippp@ere.umontreal.ca

\section{Bruce J.West}

Department of Physics

Center for Nonlinear Science

P.O. Box 305370,

Denton, TX 76203-5370

Email: bwest@fractal.phys.unt.edu

\section{Abstract}

An approach is suggested in this paper that has successfully been applied in physics, ecology, and the biomedical sciences. This is called fractal-complex-adaptive-system (FCAS) modeling. The objective of this type of analysis is to reconstruct the dynamics of the pathological process that has been leading to the disease. Diabetes, a complexdisease, has been used to test the methodology. Biometrical analyses were undertaken on subjects diagnosed with overt diabetes (hereafter called IDDM), chemical diabetes (NIDDM), and a group of normal subjects. The studied variables were plasma glucose, insulin concentration, and insulin sensitivity. FCAS modeling consists in fitting a power-law function to the bivariate lognormal distribution of the variables. The power-law exponent is estimated by principal component analysis (PCA). Analyses have shown that glucose disposal can be considered a fractal process, thereby implying a complex hierarchy of interacting scales and mechanisms in glucose handling. The first principal component represents quantitative glucose disposal, and the second component is compatible with insulin efficiency. PCA further retrieved distinct ongoing pathological processes within clinical groups of subjects. The IDDM insulin production defect had a high (absolute value) exponent of -3.5 that confirms a crude defect scanning the whole fractal hierarchy. Definite insulin resistance has been detected in clinically normal subjects with a low exponent of -0.5 , thus suggesting a subtle and complex problem possibly due to aging or reduced physical activity. Insulin sensitivity was definitely impaired in the NIDDM clinical group with an exponent of -2.2 , thereby suggesting poorly scheduled insulin feedback, possibly due to peripheral insensitivity. NIDDM appeared to result from aggravation of the subtle insensitivity seen in normal subjects. On the whole, the fractal model seemed to be capable of assessing the degree of complexity of a disease. It is concluded that future stud- 
ies of diabetes using FCAS modeling ought to be undertaken on the basis of multiplescale biological variables, thereby closely reflecting the complexity of glucose handling. It is further recommended that such analyses be undertaken with dynamic data to track down the precise timing of the various homeostatic disruptions. It would also be important to carry out this type of analysis on less known but equally complex disease processes. The results might point to important new research findings.

Keywords: Power law. Fractals. Diabetes mellitus, insulin-dependent. Diabetes mellitus, non-insulin dependent. Nonlinear dynamics. Principal component analysis. Complex-adaptive-system modeling. Allometry

\section{Resumo}

O artigo sugere uma abordagem que foi utilizada com sucesso na física, ecologia e nas ciências biomédicas. Tal abordagem recebe o nome de fractal-complex-adaptivesystem (FCAS) modeling, ou "modelagem de sistema adaptativo complexo fractal". O objetivo desse tipo de análise é reconstruir a dinâmica do processo patológico que levou à doença. O diabetes, uma doença complexa, foi utilizado para testar a metodologia. Foram realizadas análises biométricas em indivíduos diagnosticados com diabetes clínico (de agora em diante IDDM), com diabetes químico (NIDDM) e em um grupo de indivíduos normais. As variáveis estudadas foram glicose plasmática, concentração de insulina e sensibilidade à insulina. A modelagem FCAS consistia em adaptar uma função powerlaw à distribuição lognormal bivariada das variáveis. O exponente power-law é calculado através da análise do componente principal (PCA). As análises demonstraram que a disponibilidade de glicose pode ser considerada um processo fractal, sugerindo uma complexa hierarquia de escalas e mecanismos que interagem no processamento da glicose. O primeiro componente principal representa a disponibilidade em termos quantitativos de glicose e o segundo componente é compatível com a eficiência da insulina. A PCA também detectou processos patológicos distintos ocorrendo dentro dos grupos clínicos. A deficiência na produção de insulina no IDDM apresentou um alto exponente de -3.5 (valor absoluto), o que confirma uma deficiência bruta permeando toda a hierarquia fractal. Resistência permanente a insulina foi detectada em indivíduos clinicamente normais com um baixo exponente de -0.5, assim sugerindo um problema sutil e complexo, possivelmente devido ao envelhecimento ou atividade física reduzida. A sensibilidade à insulina estava permanentemente prejudicada no grupo clínico NIDDM com um exponente de -2.2, desse modo sugerindo comprometimento no 
feedback de insulina, provavelmente devido a insensibilidade periférica à insulina. $\mathrm{O}$ NIDDM parecia ser o resultado da piora da sutil insensibilidade encontrada entre os indivíduos normais. De modo geral, o modelo fractal provou ser capaz de avaliar o grau de complexidade da doença. Concluise, portanto, que estudos futuros do diabetes utilizando a modelagem FCAS devem ser realizados a partir de variáveis biológicas de escalas múltiplas, assim refletindo com exatidão a complexidade do processamento da glicose. Seria recomendável, ainda, que tais análises fossem realizadas com dados dinâmicos para investigar o momento preciso das diferentes alterações homeostáticas. Também seria importante realizar esse tipo de análise em processos patológicos menos conhecidos, mas igualmente complexos. Esse resultados poderiam levar a importantes novos achados de pesquisa.

Palavras-chave: Modelos teóricos . Fractais. Diabetes mellitus não insulinodependente. Diabetes mellitus insulinodependente. Dinâmica não linear. Análise do componente principal. Modelagem de sistema adaptativo complexo. Alometria.

\section{Introduction}

Many epidemiologists are concerned with the current difficulty in understanding health and disease. On the one hand, riskfactor modeling (e.g., multivariate logistic regression) can fail to unravel the etiology of complex disease processes ${ }^{1-3}$. For example, the small percentage of the total variation in disease incidence typically accounted for by regression analyses is generally taken as an indication of the failure of structural modeleling ${ }^{4}$. On the other hand, controversial findings are prevalent in epidemiology ${ }^{5-7}$. It is envisioned that the future of epidemiology will establish even more inconsistencies. This is expected because there will be an increased reliance on remote exposures (e.g., molecular epidemiology), the effects of which have to cross many scales of organization before producing outcomes. There are two main reasons why causal research meets with limited success nowadays ${ }^{7}$; either some crucial variables remain hidden to the investigator doing risk-factor modeling or the underlying disease dynamics results requires a complex system approach at variance with riskfactor modeling. Our contention is not that risk-factor modeling should not be used, only that new approaches based on output of complex systems with nonlinear dynamics ought to be explored. This paper looks into systems with nonlinear dynamics.

Some investigators have already suggested that recourse to nonlinear dynamics can help unravel the web of causes (the black-box) that relates exposure to disease ${ }^{2-}$ 4,8,9. A few papers have established how the presence of nonlinear dynamics in disease occurrence can, because of the non-independence of outcomes, jeopardize habitual analytical study designs ${ }^{2,3}$. Further, attempts at modeling the black-box have been made; for example, one investigator has studied a nonlinear deterministic model of the incubation period ${ }^{8}$, and neural-nets have been used for clinical pattern recognition ${ }^{10}$. These methods appear to provide new concepts and approaches for 
the early recognition of pathological processes.

In this paper, the nonlinear dynamics approach tested is called fractal-complexadaptive-system (FCAS) modeling. FCAS models have been developed by researchers from the Sante Fe Institute. Later, works related to FCAS but emphasizing their scaling or fractal nature have been carried out by a few investigators ${ }^{11-15}$. FCAS, by definition, is a hierarchy of multiple nonlinearly interrelated subsystems. FCAS models are useful whenever a phenomenon results from a signal that has been propagated through a comp lex hierarchy of scales of organization. The strength of FCAS is that it can identify the degree of smoothness or roughness of signal flow through the hierarchy. A well broken-in FCAS entails a smooth mechanism that responds in an appropriate manner to the requirements of the outer world. A defective FCAS responds selfishly, i.e., it ignores the outer intervening systems or refrains from responding smoothly to subsystems. Therefore, smoothness of response involves spread-out correlations among subsystems and feedback. Health is equated with response smoothness. Disease, on the other hand, bears various degrees of roughness (interference in signal flow). The cruder the defect, the deeper in the hierarchy the disease origin, and the more uncontrolled the mechanism (short-range or no correlations at all). Also, the deeper the disease origin, the more linear and the more obvious its relation to a putative exposure. In contradistinction, the more intricate the pathological process, the more nonlinear and the less predictable its relation to any putative exposure.

Because FCAS is complex and its components are interrelated by definition, the subsystem units and their interactions must be modeled together. Therefore, in order to assess the degree of complexity, one needs a global measure of the output of the hierarchical system. The reasoning is the following: if the output matches a given probabilistic model (specific to FCAS), then the sytem is likely complex (as defined above). This approach is in contradistinction with that of nonlinear risk-factor models such as multivariate regressions, that model the structure of interrelated observations on a nonlinear scale. The FCAS approach is not structural; it rather models the output of a complex system. Incidentally, the powerlaw function is specific to FCAS; the function quantitatively assesses the envelope of the system. The operational methodology ensuing from the above viewpoint is twofold. First, the health/disease process can be described by an evolving complex covariance structure of biological variables. Second, fractal geometry is useful in the study of biomedical phenomena with multiple scales of biological organization. The objective of this paper is to test the validity of the FCAS approach in the study of a complex disease, diabetes. The conceptual foundations of FCAS in diabetes are reviewed in Appendix I.

\section{Methods}

Fractal objects can be identified with the aid of statistics. The signature of a fractal object is a power-law function ${ }^{14,15}$. The power law describes self-similar objects/ phenomena whose mechanisms extend over many scales of observation. The power-law function is therefore a scaling law. A scaling law can be recognized in any object that has no inherent size scale (e.g., the diameter of arteries, the geometry of the lung, etc.). Self-similar hierarchical phenomena may be likened to a phylogeny with, for example, the multiple species embedded in the genera, and the genera in turn embedded in more inclusive groupings, and so on. Therefore, acknowledging a phenomenon as fractal implies similarlyorganized mechanisms over a hierarchy of scales. Such phenomena involve multiple nonlinearities; notwithstanding, they are described by a linear function under appropriate transformation of the macroscopic variables. In this sense, the power-law ressembles the logistic regression as both are nonlinear models that can be linearized. The linearization of the power-law function 
as a model for dynamics that are nonlinear should not be viewed as a defect for it has been shown that the envelope of experimental FCAS behaves that way ${ }^{15}$. The fractal dimension (D) is related to the power-law exponent, $\beta$, by the relation $\beta=3-2 D$. More specifically, the more complex the studied phenomenon, the more spread out the correlations among scales.

In physical phenomena the power-law exponent of the power spectrum is often negative (inverse power law). The power-law exponent is an accurate assessment of the dispersion of the distribution ${ }^{14,15}$. A small power-law exponent, between 0 and -2, refers to large dispersion and high complexity (no characteristic scale). A smaller powerlaw exponent indicates lower dispersion and points to a more straightforward phenomenon. In other words, the smaller the inverse power-law exponent, the simpler, the more predictable, the more regular, and the more easily boxed-in is the process. These phenomena can successfully be investigated by linear methods. Contrariwise, the larger the value of the negative power-law exponent, the more complex, the more intricate, and the less easily boxed-in is the process. Linear methods are useless here. This is the domain of nonlinear dynamics. A power-law exponent of zero stands for white noise processes, i.e., chance phenomena with utter unpredictability. The mathematical underpinnings of the principal component analysis (PCA) and the power law are detailed in Appendix II.

\section{Caveats}

Before embarking on the analysis per se, four issues deserve comment. First, diabetes can be considered a complex multiplescale phenomenon. Accordingly, a crosssectional sample of plasma measures of sufficient variability will embody the various scales of organization of the glucose regulation process of different subjects. Therefore, a cross-section of subjects from the same disease stage will reflect the complex dynamics of the latest pathological event. One can hope to reconstruct the dy- namics of the latest pathological phase transition if it can be assumed that the measurement-specific biological variability is extended enough to cover the recent history of the disease process. More simply put, one uses a cross-section of various individuals as a surrogate for the individual disease pathway. In this context, the exponent of the allometric law becomes the expression for an observation of self-similarity in a series of fractally structured individuals ${ }^{21}$.

Second, structural relationships of healthy phenomena are generally measured by a positive power-law exponent. Accordingly, the disease state should be probed by a qualitative change in the covariance structure with respect to normalcy. Hence, the structure of a pathological process will translate to an "involuted" covariance structure leading to the reverse patterning of healthy relationships, and its measure will be an inverse power law.

The third issue to make clear is that PCA will break down the covariance structure into independent variables or dimensions, each having its own power-law exponent. This will occur if multiple sources (two here) of variation coexist. In the latter case, the second principal component, orthogonal to the first, will necessarily yield at least one inverse power law. But, of course, nothing prevents only one component from being significant. Obtaining two significant principal components will point to biological heterogeneity.

Last, it must be stressed that the main thrust of this paper is not to reduce the dimensions of the data, but to provide values akin to the fractal dimensions of the processes involved in the data. While the method we use to achieve this aim is actually a PCA, the latter should be viewed simply as a mean for increasing the validity of the measures of the computed fractal dimensions.

\section{Material}

This study uses Reaven and Miller's data $\operatorname{set}^{23}$. The publis hed data were easily acces- 
sible $^{24}$ and composed of 145 subjects. The subjects were given an oral glucose tolerance test (OGTT) with blood samples drawn during the subsequent three hours for measurement of plasma glucose and immunoreactive insulin concentrations. Only one measurement was obtained per subject. The variables studied were glucose area, insulin area, and steady state plasma glucose. This latter variable measures the ability of different subjects to dispose of identical glucose loads under the same insulin stimulus and is therefore considered a marker of insulin efficiency. Subjects were classified into three groups according to the OGTT results. 33 of the subjects had overt diabetes (abnormal fasting levels), 36 had chemical diabetes (normal fasting levels with abnormal GTT response), and 76 were considered normal. None of the subjects were receiving insulin or oral hypoglycemics at the time of diagnosis. The subjects were non-obese. Subjects' age and weight were not correlated with the above three variables. The details of the protocols and diagnostic criteria can be found in Reaven \& Miller $^{23}$. For convenience reasons, the three diagnostic groups are hereafter called the IDDM (overt diabetes), NIDDM (chemical diabetes), and normal group.

\section{Results}

Figure 1 displays the bivariate scattergrams of the original values of glycemia by insulin output and of insulin output by insulin sensitivity for the 145 patients. Only these two relationships are taken up here. Figure 2 displays the same bivariate relationships according to the logs of the observations. The three clinical groups of subjects appear well surrounded by $95 \%$ equiprobable ellipses, thereby suggesting bivariate lognormality. The good fit suggests that the fractal model can describe glucose disposal in all three clinical groups of subjects. Ellipse major axes are also drawn in Figure 2; the major axes are the power laws of the first PCA (to be presented below). In order not to clutter Figure 2, the power laws of the second PCA (perpendicular to the major axes) have not been sketched out.

Tables 1, 2, and 3 set out PCA results. Table 4 displays power-law exponents. Only the first two components are shown because their contribution to variability amounts to nearly the total variance. Table 1 suggests that the group of normal subjects is heterogeneous with $73 \%$ of the variance explained by the first component. This
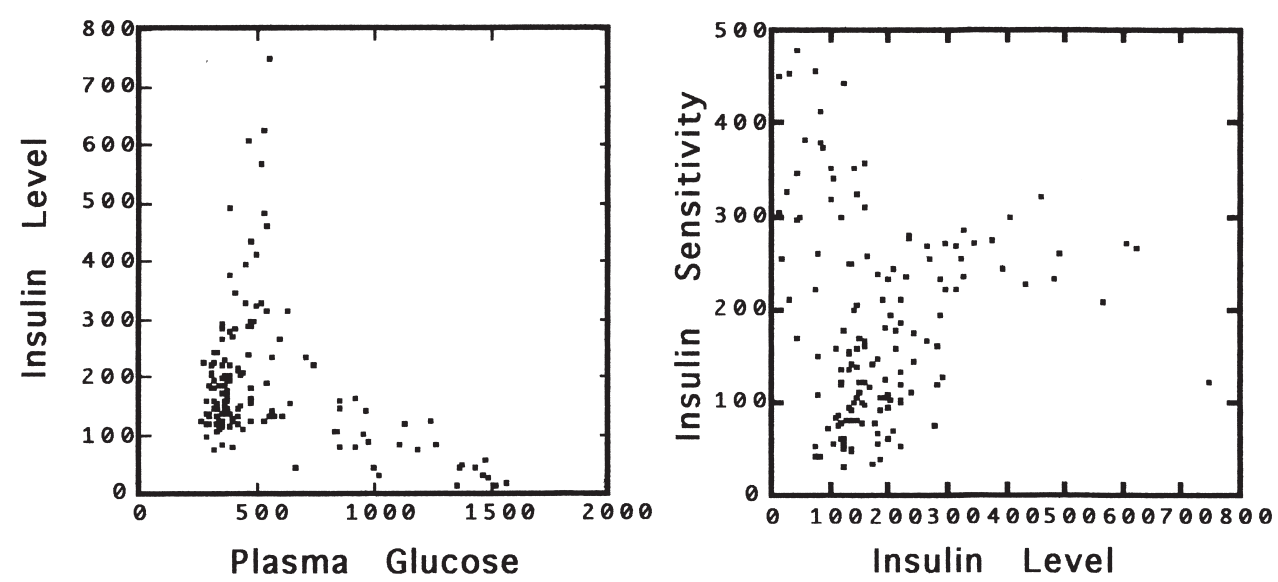

Figure 1 - Scattergram of plasma glucose $(\mathrm{mg} / 100 \mathrm{ml} / \mathrm{hr})$ by insulin concentration $(\mu \mathrm{U} / \mathrm{ml} / \mathrm{hr})$ : Original data 

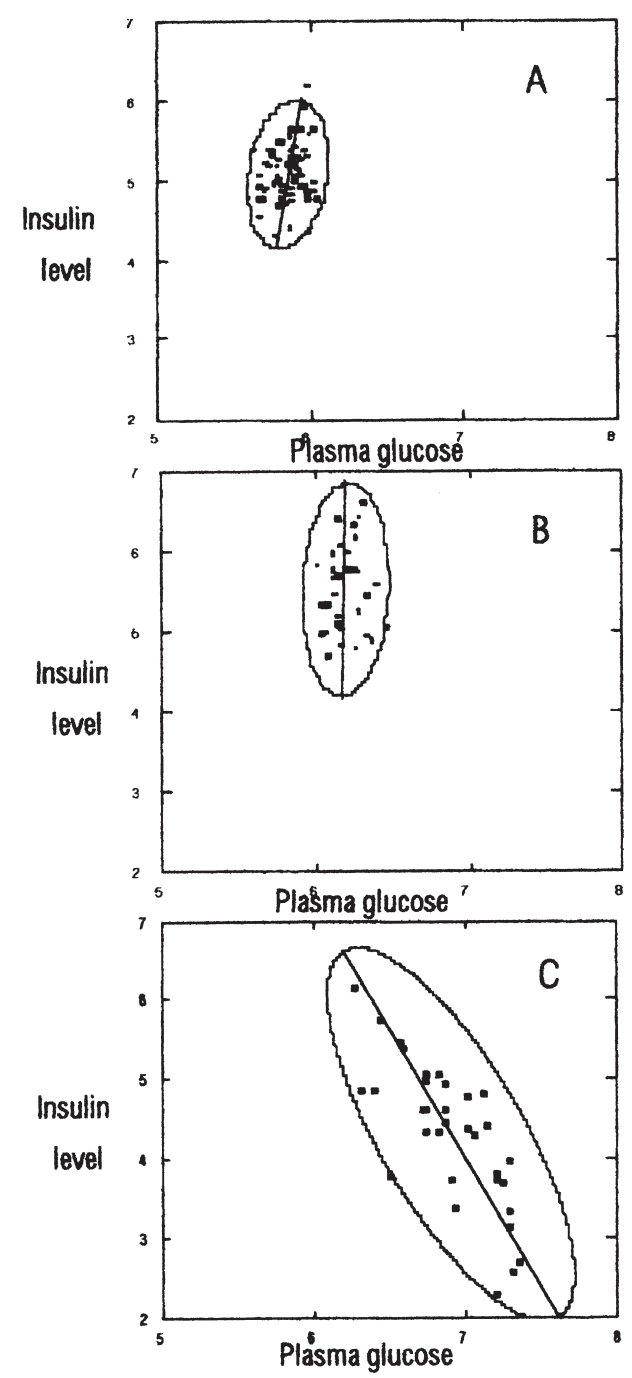

Figure 2 - Scattergram of plasma glucose $(\mathrm{mg} / 100 \mathrm{ml} / \mathrm{hr}$ ) by insulin concentration $(\mu \mathrm{U} /$ $\mathrm{ml} / \mathrm{hr}$ ) in normal subjects (A), NIDDM patients (B) and IDDM patients (C): Log data, power laws, and $95 \%$ ellipse contours

component is dominated by insulin sensitivity and concentration. It shows that a glucose load does not elicit a high concentration of plasma glucose.

Insulin concentration, however, is proportional to power 9 of plasma glucose (Table 4), and sensitivity increases according to power 2 of insulin concentration. To fix ideas, a proportional increase of power 1 would mean equal increase over any two variables. In the case of the glucose and insulin concentration of normal subjects, the result shows that a unit increase in glucose entails a nonlinear reaction of insulin with the latter increasing as power 9 of glucose. As we shall see below, this power curve is far less steeper than that found in NIDDM subjects. Because these results pertain to presumably normal subjects, the results can be considered the gold standard against which findings on diseased subjects ought to be assessed. The first component therefore describes how normal subjects quantitatively dispose of a glucose load.

The second component of the normal group is dominated by the contrast of insulin concentration and sensitivity. Sensitivity decreases very slowly according to a power of -0.5, with an increase in insulin concentration. This component of variation is not negligible (accounting for $24 \%$ of the total variance). It describes how clinically normal subjects qualitatively dispose of a glucose load. The inverse power law indicates that this is a source of pathological variation.

The results of the analysis of the data obtained from the subjects diagnosed with NIDDM are presented in Table 2 . The analyses shown in Table 4 indicate that, in these subjects, glucose disposal (first component) required very high concentrations of insulin $(\beta=82.7)$ and that sensitivity is low

Table 1 - PCA component loadings in clinically normal subjects.

\begin{tabular}{lccc}
\hline & PRINCIPAL COMPONENTS \\
& 1 & 2 & 3 \\
Plasma glucose & 0.026 & 0.008 & -0.103 \\
Insulin concentration & 0.239 & 0.281 & 0.005 \\
Insulin sensitivity & 0.496 & -0.136 & 0.003
\end{tabular}

VARIANCE EXPLAINED BY COMPONENTS

$\begin{array}{ccc}1 & 2 & 3 \\ 0.304 & 0.098 & 0.011\end{array}$

PERCENT OF TOTAL VARIANCE EXPLAINED

\begin{tabular}{rrr}
1 & 2 & 3 \\
73.6 & 23.7 & 2.7 \\
\hline
\end{tabular}


Table 2 - PCA component loadings in clinically diagnosed NIDDM subjects.

\begin{tabular}{lccc}
\hline & \multicolumn{3}{c}{ PRINCIPAL COMPONENTS } \\
& 1 & 2 & 3 \\
Plasma glucose & 0.006 & 0.017 & 0.108 \\
Insulin concentration & 0.496 & 0.126 & -0.004 \\
Insulin sensitivity & 0.236 & -0.264 & 0.005
\end{tabular}

VARIANCE EXPLAINED BY COMPONENTS

$$
\begin{array}{ccc}
1 & 2 & 3 \\
0.302 & 0.086 & 0.012
\end{array}
$$

PERCENT OF TOTAL VARIANCE EXPLAINED

\begin{tabular}{rrr}
1 & 2 & 3 \\
75.6 & 21.5 & 2.9 \\
\hline
\end{tabular}

Table 3 - PCA component loadings in clinicaliy diagnosed IDDM subjects.

\begin{tabular}{lccc}
\hline & \multicolumn{3}{c}{ PRINCIPAL COMPONENTS } \\
& 1 & 2 & 3 \\
Plasma glucose & 0.249 & 0.151 & 0.110 \\
Insulin concentration & -0.895 & 0.065 & 0.026 \\
Insulin sensitivity & 0.071 & 0.292 & -0.062
\end{tabular}

VARIANCE EXPLAINED BY COMPONENTS

$$
\begin{array}{ccc}
1 & 2 & 3 \\
0.868 & 0.112 & 0.017
\end{array}
$$

\begin{tabular}{|c|c|c|}
\hline & \multicolumn{2}{|c|}{ Clinically normal subjects } \\
\hline & Insulin $=*$ Sensitivity ${ }^{\mathrm{b} * *}$ & Glucose $=$ Insulin ${ }^{b}$ \\
\hline First component & 2.1 & 9.2 \\
\hline \multirow[t]{3}{*}{ Second component } & -0.5 & 35.1 \\
\hline & \multicolumn{2}{|c|}{ Clinically diagnosed NIDDM subjects } \\
\hline & Insulin = Sensitivity ${ }^{b}$ & Giucose $=$ Insulin \\
\hline First component & 0.5 & 82.7 \\
\hline \multirow[t]{3}{*}{ Second component } & -2.1 & 7.4 \\
\hline & \multicolumn{2}{|c|}{ Clinicaliy diagnosed IDDM subjects } \\
\hline & Insulin = Sensitivity ${ }^{\mathrm{b}}$ & Glucose $=$ Insulin ${ }^{b}$ \\
\hline First component & -0.08 & -3.5 \\
\hline Second component & 4.5 & 0.4 \\
\hline
\end{tabular}

PERCENT OF TOTAL VARIANCE EXPLAINED

\begin{tabular}{crr}
1 & 2 & 3 \\
87.1 & 11.2 & 1.7 \\
\hline
\end{tabular}

Table 4 - Power law exponents estimated over the first two principal components.

* The parameter a of the power law has been discarded since it is not interpreted.

${ }^{* *} \mathrm{~b}$ is the power law exponent.

( $(=0.5)$ in comparison with normal subjects (Table 1 first component). On the other hand, accounting for $21 \%$ of the variance, the second component indicates an important decrease in sensitivity $(\Omega=-2.1)$ with low insulin output. The second component points to a source of pathological variation similar to but more severe than of the clinically normal subjects (Table 1 second component).

The results of the analysis of the data obtained from the subjects diagnosed with IDDM are presented in Table 3. This group is more homogeneous, where the first component accounts for $87 \%$ of the total variation. The analysis shown in Table 4 suggests an abrupt decrease in insulin output when plasma glucose concentration was high (Table 3). The second component, which represents a low source of total variation (11\%), shows low insulin output but very high sensitivity to insulin. 


\section{Discussion}

The methodology used in this paper is not new. The allometry law is often found in biology. PCA is ubiquitous in science, and fractals are common in physics. What is new is the unification of fractal modeling, allometric law, and the principal components in the framework of a coherent methodology intended to understand the nonlinear dynamics (by FCAS) of diabetes.

These analyses suggest that glucose disposal can be considered a FCAS. This conclusion is supported in normal, NIDDM, and IDDM subjects. The conclusion is based on a good log-log fit of the three biological variables (plasma glucose, insulin concentration, and insulin efficiency) to a power law. Identifying a system as FCAS leads to invoking a fractal process in diabetes, i.e., to stating that glucose management is controlled by amplification ${ }^{12}$. This statement means that there must be secondary mechanisms of glucose disposal besides insulin. These mechanisms are leverage mechanisms. Leverage can be understood in the following way. Suppose one wishes to work more efficiently. What is this person expected to do? He/she has two choices. First, work more, i.e., work during the evenings, the week-ends, etc. Such a regimen eventually leads to exhaustion. If overwhelmed by work, the person also has a second choice, i.e., hire a team that will work "under" him/her and according to his/her needs. This is a leverage mechanism. Further, nothing prevents the team members from sharing the work with others, hired on an irregular basis. Amplification, a characteristic of fractal processes, has the virtue of fine-tuning complex processes that need constant and subtle adjusting to challenging environmental conditions ${ }^{11,12}$. Studies on diabetes confirm the presence of more than one mechanism of glucose disposal in humans ${ }^{25}$. There are redundant glucoregulatory factors, and a hierarchy exists among them. Although insulin stands at the top of the hierarchy, glucoregulation is not achieved by insulin alone.
The use of PCA has identified two independent components of variation in each clinical group. One component is associated with quantitative glucose disposal and the other with insulin efficiency. Subjects with IDDM had large loadings on the first component, and those with NIDDM correlated strongly with the second component. According to this distinction, IDDM is clearly different from NIDDM. Whereas IDDM is a defect of insulin output, NIDDM is a defect of insulin efficiency. It was intriguing to observe that normal subjects also loaded lightly on the second component.

The use of PCA also resulted in the identification of distinct pathological processes within clinical groups, thereby reflecting the involution of the covariance structure. Covariance structure involution was observed when the power-law exponent turned from a positive to a negative value. This change is based on the assumption that healthy biological processing implies positive relationships among variables. This statement is true for glucose disposal. Other biological processes might behave differently.

That IDDM is a defect of insulin production was dramatically confirmed by its severity in the clinically IDDM group. The very small power-law exponent of -3.5 supports the well-known contention of a straightforward defect in insulin production. Although few mechanisms are expected at as high a scale of organization, the very small exponent does not rule out more than one origin to the defect. According to the covariance structure, IDDM is not preceded by a pre-diabetic state; IDDM therefore results from an abrupt change within a very short incubation period. This result is consistent with the usually precocious age of onset of the condition. Such a small exponent suggests a lock-in process with no adaptability. A likely explanation is that a potent initial condition has drastically bypassed the nonlinear intricacies of the glucose handling hierarchy. This explanation further suggests that simple insulin therapy can scarcely deal with hyperglycemia except as a palliative intervention. For therapy to 
mimic the intrinsic complexity of the glucose handling process of normal subjects, a much smoother intervention would be required. As a result, current insulin therapy is only expected to crudely control hyperglycemia. Besides, the insulin-sensitivity-toproduction ratio has a nearly null exponent $(-.08)$. The latter exponent suggests that insulin sensitivity is left uncontrolled in IDDM patients. The latter conclusion would be consistent with a basic pleiotropic defect.

The NIDDM clinical group also supports the quantitative-glucose-disposal interpretation of the first principal component. This finding suggests that NIDDM patients have hyperinsulinemia following a glucose load. The most popular interpretation of this relates to lower than normal insulin sensitivity, i.e., insulin resistance. This hypothesis is not contradicted by the results. A competing hypothesis would be that hyperinsulinemia leads to low efficiency; this can occur if insulin becomes insensitive after a threshold level of insulin secretion is exceeded. A first mechanism explaining the latter hypothesis might be a defect in insulin structure; a second mechanism would be impaired insulin bioreactivity. The latter hypothesis is in line with the IDDM second component according to which very low insulin output accompanies very high insulin sensitivity.

The second principal component relates to insulin efficiency. Definite insulin resistance is noted in the clinically normal and NIDDM groups. The sensitivity problem is not dramatic in clinically normal subjects who are (and may remain) symptomless. This might refer to a subtle aging effect. The exponent of -0.5 points to a highly complex mechanisms of glucose regulation. This exponent also implies that the insulin insensitivity of the normal subjects is due to complex interactions among several finelytuned mechanisms.

Insulin sensitivity is definitely impaired in the NIDDM clinical group. The defect is more severe than that of normal subjects. The defect, with a power-law exponent of 2.1 is crude, and can be located deep in the hierarchy of insulin-sensitivity control achievement. It suggests a well-delineated clinical picture consistent with a potent genetic initial condition that can bypass the complex dynamics' nonlinearities of the pathological process. More often than not complex nonlinearities will prevent any easily-recognized one-to-one relationship between cause and effect in disease processes. Further, the small exponent suggests a persistence effect, i.e., lack of control due to poorly scheduled feedbacks. Such a high exponent is in contradistinction with the swift response of normal subjects to a glucose challenge. The persistence effect might therefore coincide with a lower-than-normal peripheral sensitivity to insulin. This explanation is in line with a post-receptor defect in peripheral tissues and hence with poor negative insulin feedback ${ }^{26}$.

The NIDDM persistence effect is quite crude, thus preventing any capacity of complex and finely-tuned regulation. Further, insulin efficiency proved to be lower-thanoptimal in normal subjects; this suggests a lengthy pre-diabetic process to the NIDDM clinical state. This suggestion is not at variance with a genetic hypothesis but nevertheless more in line with a long-run (continuous) environmental and/or behavioral effect. The reducing of physical activity with age is a likely intervening event. The latter considerations mean that normal glucose regulation is controlled by swift reaction times due to fine-tuning by the glucoseregulation control hierarchy. The latter control is lost in NIDDM. All the above results are consistent with the most recent research results on diabetes ${ }^{26,27}$.

The hypothesis that both principal components revealed two different aspects of the organism response to a glucose load (i.e., insulin production and insulin efficiency) has been discussed. Nonetheless, an alternative interpretation is also possible: the principal components might represent subgroups' variation, i.e., etiologic heterogeneity. According to this viewpoint, the first component of the clinically normal group clearly outlines the normal condition. The 
second component would represent undiagnosed but precocious cases of NIDDM. This pre-diabetic condition would evolve to a full-blown NIDDM dynamical process as exemplified by the NIDDM group secondprincipal-component. Similarly, the first component of the NIDDM group would describe subjects having a normal response to a glucose load, i.e., simple hyperinsulinemia with lower yet normal insulin sensitivity. These subjects would incorrectly be considered NIDDM patients. Likewise, the clinical IDDM group would be composed of two subgroups. The first component would point to the most common IDDM type with autoimmune insulin destruction. The second component would refer to subjects with unusually high glucose concentrations, very low insulin output, but very high insulin efficiency. These normal subjects would presumably be those with incipient beta-cell destruction $^{28}$.

This epidemiologic study of a complex disease is the first to make use of FCAS modeling. For a first study, methodological and interpretative aspects have been emphasized. Further, to test the new methodology, the analysis has been carried out on a complex but well-studied disease process. Cross-sectional data such as those used here are easy to gather and they can yield important clues as to the disease process. Purely dynamical data allowing for multiple repeated measurements would nonetheless be the most useful approach in unravelling the various steps of the pathological progression. Though this cross-sectional analysis cannot definitely distinguish between a disease's natural history and subgroups' heterogeneity, it is surmised that this type of data reveals a dynamic viewpoint, for the power law describes how biologic variables change in different subjects. Our results are in line with what is known of the biology of diabetes, and agrees well with past applications of the method to other complex biological processes. This gives credence to the validity of the methodology used. The FCAS model can assess the complexity of a disease process. FCAS does not evaluate cau- sality. In no way the computed the powerlaw exponents (or FDs to be calculated therefrom) are indices or arguments on behalf of causality. To be specific, FCAS provides an assessment of the complexity of the dynamics of the process. Furthermore, this study suggests new directions of research. Future diabetes studies using fractal modeling ought to be undertaken with biological components (e.g., glucagon) reflecting various scales in the hierarchy of glucose handling. It would also be important to carry out this type of analysis on less known but equally complex disease processes. The results could uncover unusual findings and provide clues to further research.

\section{Acknowledgements}

We are particularly indebted to Nick Birkitt, Michael J. Glade, Elizabeth Lin, Will Maier, and Dena Schanzer for many helpful comments and suggestions. We are also grateful to Katherine Frohlich and Ron Levy for a review of the manuscript style.

\section{APPENDIX I}

\section{CONCEPTUAL UNDERPINNINGS OF FCAS}

FCAS, when applied to health and disease, proposes to replace the concept of states with that of processes. Second, the approach considers disease as an emergent property resulting from a late, abrupt, and unsuccessful attempt of the organism to maintain homeostasis ${ }^{16}$. In the context of FCAS, precisely because complex diseases imply emerging behaviors, "homeodynamics" is a more appropriate term than homeostasis $^{30}$. The latest property of the disease process is one among several emergent properties that the organism has experienced over pathological time and across the hierarchy of scales of organization. This viewpoint implies that the clinical disease is the result of multiple critical threshold crossings (or phase transitions as when, upon consistent freezing, water mol- 
ecules self-organized into ice crystals) induced by far-from-equilibrium dynamics, with irreversibility as the endpoint ${ }^{17}$. Disequilibrium is conceived as a dynamic state which includes the seeking of solutions to environmental aggression. This definition of disease further implies that the pathological process cannot be considered separate from the health process ${ }^{18}$. One therefore has to model health and disease processes together. A remarkable expository paper of possible FCAS in hypertension is Weder \& Schork $^{19}$.

Considering health and disease as a complex dynamic has two important consequences. First, clinical disease is the latest of the emergent properties of the gradual dynamic unfolding of the subject's historical events. This memory-keeping but incessantly reorganizing complex structure is based on interrelated events. Second, inputs to this process are shared by several different interacting scales of biological organization involving feedback. The overall result of this complex parallel processing is information translation and specificity loss. Therefore, any clinically observable property is a process endpoint whose causes cannot be linearly determined. A possible metaphor for the pathological process is a walk in a maze, the walls of which rearrange themselves with every step ${ }^{20}$.

\section{APPENDIX II}

\section{MATHEMATICAL UNDERPINNINGS OF FRACTAL MODELING}

The inverse power law is a hyperbolic function with no characteristic scale and, therefore, infinite moments. In particular, its theoretically infinite variance provides space over all scales of organization for unexpected emergent events. The positive power law is given by: $\mathrm{Y}=\mathrm{a} \mathrm{X}^{\mathrm{B}}$ where $\mathrm{a}$ is a parameter, $\mathrm{X}$ and $\mathrm{Y}$ are two intercorrelated biological variables, and $\beta$ is the power-law exponent. In biology, this equation is known as the allometric law ${ }^{21}$. The exponent of the power law is usually positive. It describes the differential rate of change of two variables from a cross-sectional survey of organisms. When fitted to the bivariate relationship of variables on log-log graph paper, the function yields a straight line.

A covariance structure refers to a set of multiple interrelated variables. To deal with multiple variables does not detract from using the power law. Incidentally, the fitting procedure through the major axes generalizes easily to multiple variables through principal component analysis (PCA) ${ }^{22}$.

The power-law exponent can be estimated in many ways. In physics, it is usually estimated by least squares ${ }^{15}$. This poses a unique problem for biological variables since no dependent variable is implied by the concept of covariance structure. Further, the $\mathrm{X}$ and $\mathrm{Y}$ variables both involve measurement errors. The best way to deal with this situation is to estimate the slope of the major axis (instead of any of the regression lines). Accordingly, neither the $\mathrm{X}$ nor the $\mathrm{Y}$ variable should be privileged by the fitting procedure: the minimization should then be undertaken orthogonally with respect to the major axis instead of vertically or horizontally with respect to regression lines. Doing so, however, involves one further difficulty: the slope of the major axis is attracted towards the variable with the largest measurement error or the largest scale. One then has to take the logarithm of the variables to stabilize the variance or render the slope estimate scale-invariant under nonlinear scale changes ${ }^{22,29}$.

Seeking the principal components of the covariance matrix of the log variables of a bivariate swarm of points yields a matrix of loadings, the first vector of which represents the direction cosines of the major axis and the second vector, the direction cosines of the minor axis such that:

$$
\begin{aligned}
& \mathrm{Y} 1=\left[\begin{array}{ll}
\cos \mathrm{t} & \sin \mathrm{t}
\end{array}\right][\mathrm{X} 1-\mu 1] \\
& \mathrm{Y} 2=\left[\begin{array}{ll}
-\sin \mathrm{t} & \cos \mathrm{t}][\mathrm{X} 2-\mu 2]
\end{array}\right.
\end{aligned}
$$

where $\mathrm{Y} 1$ and $\mathrm{Y} 2$ are the new coordinate axes (the principal components), cos $t$ and $\sin t$ 
are the direction cosines of the rotation to the new axes, and the [Xi - $\mu \mathrm{i}]$ correspond to the translation with respect to the original coordinate axes undertaken by the PCA. The $\mu \mathrm{i}$ are the means of the original $\mathrm{Xi}$ variables. The above yields:

$$
\{\mathrm{Y} / \mathrm{Gy}\}^{1 / \cos \mathrm{t}}=\{\mathrm{X} / \mathrm{Gx}\}^{1 / \sin \mathrm{t}}
$$

where: $\mathrm{Y}$ and $\mathrm{X}$ are the arithmetic values; Gy and Gx are the geometric means of $\mathrm{Y}$ and $\mathrm{X}$;

and $\cos t$ and $\sin t$ are the direction cosines of the matrix of factor loadings of the logarithmic covariance matrix.

It results from the above that:

$Y=\left\{G y / G x^{(\cos t / \sin t)}\right\} X^{(\cos t / \sin t)}$

and therefrom: $\mathrm{Y}=\mathrm{a} \mathrm{X}^{\beta}$

Therefore, the above transforms back to the power law, yielding the exponent $\beta$ as the ratio of the direction cosines of the bi- variate swarm of points. Compared to estimating through least squares, there are three advantages to the above procedure. First, the slope of the power law is biologically meaningful because it specifies a structure rather than a dependence relationship. Second, one obtains not only the value of the slope but also the estimates of differential change in both variables. Third, one can straightforwardly assess the complexity of the dynamics from a simple assessment of the power-law exponent.

Obtaining the slopes of the orthogonal axes of a high-dimensional swarm of points is a simple matter of performing a PCA on the covariance matrix of the logged data. More specifically, the matrix of factor loadings then yields the direction cosines of all the axes of the hyperellipsoid. The direction cosines can then be used to estimate the various power-law slopes. To use principal components on the logged data of the covariance matrix, one must ensure that the swarm of points is normally distributed; this can be assessed by a $95 \%$ equiprobable ellipse contour on each pair of variables ${ }^{29}$.

\section{References}

1. Krieger N. Epidemiology and the web of causation: Has anyone seen the spider? Soc Sci Med 1994; 39: 887-903.

2. Halloran ME, Struchiner CJ. Study designs for dependent happenings. Epidemiology 1991; 2: 331-8.

3. Koopman JS, Longini Jr, IM. The ecological effects of individual exposures and nonlinear disease dynamics in populations. Am J Publ Health 1994; 84: 836-42.

4. Philippe P. Chaos, population biology, and epidemiology. Some research implications. Hum Biol 1993; 65: 525-46.

5. Horwitz RI, Feinstein AR. Methodologic standards and contradictory results in FCASe-control research. Am J Med 1979; 66: 556-84.

6. Feinstein AR. Scientific standards in epidemiologic studies of the menace of daily life. Science 1988; 242 : 1257-63.

7. Taubes G. Epidemiology faces its limits. Science 1995; 269: $164-9$
8. Philippe P. Sartwell's incubation period model revisited in the light of dynamic modeling. J Clin Epidemiol 1994; 47: 419-33.

9. May RM. Nonlinearities and complex behavior in simple ecological and epidemiological models. Ann NY Acad Sci 1987; 504: 1-15.

10. Baxt WG. Complexity, chaos and human physiology: The justification for non-linear neural computational analysis. Cancer Lett 1994; 77: 85-93.

11. Montroll EW, Shlesinger MF. On 1/f noise and other distributions with long tails. Proc Natl Acad Sci USA 1982; 79: 3380-3.

12. West BJ, Shlesinger MF. The noise in natural phenomena. Am Sci 1990; 78: 40-5.

13. Bak P, Chen K. Self-organized criticality. Sci Am 1991 Jan; 264: 46-53.

14. Schroeder M. Fractals, chaos, power laws. New York: Freeman; 1991. 
15. West JB, Deering WD. The lure of modern science: Fractal thinking. World Scientific Publishing; 1995.

16. Sing CF, Zerba KE, Reilly SL. Traversing the biological complexity in the hierarchy between genome and CAD (coronary artery disease) endpoints in the population at large. Clin Genet 1994; 6: 6-14.

17. Yates FE. Order and complexity in dynamical systems. Homeodynamics as a generalized mechanisms for biology. Math Comput Modelling 1994; 19: 49-74.

18. Firth WJ. Chaos - predicting the unpredictable. Br Med J 1991; 303: 1565-8.

19. Weder AB, Schork NJ. Adaptation, allometry, and hypertension. Hypertension 1994; 24: 145-56.

20. Baum F. Researching public health: Behind the qualitative-quantitative methodological debate. Soc Sci Med 1995; 40: 459-68.

21. Sernetz M, Gelleri B, Hofmann J. The organism as bioreactor. Interpretation of the reduction law of metabolism in terms of heterogeneous catalysis and fractal structure. J Theoret Biol 1985; 117: 209-30.

22. Jolicoeur P. The multivariate generalization of the allometry equation. Biometrics 1963; 19: 497-9.
23. Reaven GM, Miller RG. An attempt to define the nature of chemical diabetes using a multidimensional analysis. Diabetologia 1979; 16: 17-24.

24. Andrews DF, Herzberg AM. Data. A collection of problems from many fields for the student and research worker. New York: Springer-Verlag; 1985.

25. Cryer PE. Regulation of glucose metabolism in man. J Intern Med Supplement 1991;735: 31-9

26. Yki-Jarvinen M. Pathogenesis of non-insulin-dependent diabetes mellitus. Lancet 1994; 343: 91-4.

27. Stout RW. Glucose tolerance and ageing. J R Soc Med 1994; 87: 608-9.

28. Ciampi A, Schiffrin A, Thiffault J, Quintal H, Weitzner G, Poussier P, Lalla D. Cluster analysis of an insulinodependent diabetic cohort towards the definition of clinical subtypes. J Clin Epidemiol 1990; 43: 701-15.

29. Jolicoeur P, Heusner A. The allometry equation in the analysis of the standard oxygen consumption and body weight. Biometrics 1971; 27: 841-55.

30. West BJ. Fractal physiology: A paradigm for adaptive response. In: Puermatikas ST, editor. Singular behavior and nonlinear dynamics. Singapore: World Scientific; 1989. p. 643-64. 